\title{
Cloroquina y sus derivados en el manejo de la COVID-19: una revisión sistemática exploratoria
}

\author{
Juan Pimentel ${ }^{1,2,3}$, Neil Andersson ${ }^{1,4}$ \\ ${ }^{1}$ Community Information and Epidemiological Technologies - Participatory Research at McGill, \\ CIET-PRAM, Department of Family Medicine, McGill University, Montreal, Canada \\ ${ }^{2}$ Facultad de Medicina, Universidad de La Sabana, Chía, Colombia \\ ${ }^{3}$ Escuela de Medicina y Ciencias de la Salud, Universidad del Rosario, Bogotá, D.C., Colombia \\ ${ }^{4}$ Centro de Investigación de Enfermedades Tropicales, Universidad Autónoma de Guerrero, \\ Acapulco, México
}

Introducción. Recientemente, investigadores chinos y franceses reportaron la eficacia de la cloroquina y la hidroxicloroquina para inhibir la replicación in vitro del virus SARS-CoV-2. La diseminación oportuna de la información científica es clave en tiempos de pandemia. Es urgente contar con una revisión sistemática sobre el efecto y la seguridad de estos medicamentos en la COVID-19.

Objetivo. Describir el estado actual de la literatura científica publicada hasta el 25 de marzo de 2020 sobre el uso de la cloroquina o sus derivados en el manejo de pacientes con COVID-19.

Materiales y métodos. Se hizo una revisión sistemática exploratoria en PubMed, Embase, Lilacs y 15 bases de datos de la Plataforma de Registros Internacionales de Ensayos Clínicos de la Organización Mundial de la Salud (OMS). Se incluyeron publicaciones empíricas y teóricas en inglés, español, italiano, francés o portugués, y se hizo una síntesis narrativa de los resultados.

Resultados. Se incluyeron 19 documentos y 24 registros de ensayos clínicos $(n=43)$ de 18.059 pacientes. El $66 \%$ (16/24) de los ensayos están registrados en China. Nueve ensayos evalúan la cloroquina exclusivamente y ocho, la hidroxicloroquina. Los documentos son comentarios $(n=9)$, estudios in vitro $(n=3)$, revisiones narrativas $(n=2)$, guías de práctica clínica $(\mathrm{n}=2)$, así como una revisión sistemática, un consenso de expertos y un ensayo controlado. Conclusiones. Un ensayo clínico pequeño $(n=26)$, no aleatorizado y defectuoso, respalda el uso de la hidroxicloroquina en pacientes con COVID-19. Se requiere de manera urgente tener acceso a los resultados de otros ensayos clínicos para determinar la efectividad y la seguridad de la cloroquina y sus derivados en pacientes con COVID-19.

Recibido: 29/03/2020

Aceptado: $07 / 04 / 2020$

Publicado: 09/04/2020

\section{Citación:}

Pimentel J, Andersson N. Cloroquina y sus derivados en el manejo de la COVID-19: una revisión sistemática exploratoria. Biomédica. 2020;40(Supl.2):80-95.

https://doi.org/10.7705/biomedica.5478

\section{Correspondencia:}

Juan Pimentel, Centro de Investigación de Community Information and Epidemiological Technologies - Participatory Research at McGill, CIET-PRAM, Department of Family Medicine, McGill University, 5858 Chemin de la Côte-des-Neiges, 3rd floor, Montreal H3S 1Z1, Quebec, Canada Teléfono: (+1) (514) 3987375 juan.pimentel@mail.mcgill.ca

\section{Contribución de los autores:}

Juan Pimentel: diseño del estudio

Neil Andersson: interpretación de los resultados

Ambos autores participaron en la recolección y

análisis de los datos y en la escritura del manuscrito.

\section{Financiación:}

Ninguna

Conflicto de intereses:

Los autores declaran que no tienen ningún conflicto de intereses.
Palabras clave: cloroquina; hidroxicloroquina; coronavirus; revisión sistemática; ensayo clínico; pandemias; SARS-CoV-2.

\section{Chloroquine and its derivatives in the management of COVID-19: A scoping review}

Introduction: Recently, researchers from China and France reported on the effectiveness of chloroquine and hydroxychloroquine for the inhibition of SARS-CoV-2 viral replication in vitro. Timely dissemination of scientific information is key in times of pandemic. A systematic review of the effect and safety of these drugs on COVID-19 is urgently needed.

Objective: To map published studies until March 25, 2020, on the use of chloroquine and its derivates in patients with COVID-19.

Materials and methods: We searched on PubMed, Embase, Lilacs, and 15 registries from the World Health Organization's International Clinical Trials Registry Platform for theoretical and empirical research in English, Spanish, Italian, French, or Portuguese until March 25, 2020, and made a narrative synthesis of the results.

Results: We included 19 records and 24 trial registries $(n=43)$ including 18,059 patients. China registered $66 \%(16 / 24)$ of the trials. Nine trials evaluate chloroquine exclusively and eight hydroxychloroquine. The records are comments $(n=9)$, in vitro studies $(n=3)$, narrative reviews $(n=2)$, clinical guidelines $(n=2)$, as well as a systematic review, an expert consensus, and a clinical trial.

Conclusions: One small $(n=26)$, non-randomized, and flawed clinical trial supports hydroxychloroquine use in patients with COVID-19. There is an urgent need for more clinical trial results to determine the effect and safety of chloroquine and hydroxychloroquine on COVID-19.

Keywords: Chloroquine; hydroxychloroquine; coronavirus; systematic review; clinical trial; pandemics; SARS-CoV-2. 
El 12 de marzo de 2020, la Organización Mundial de la Salud (OMS) declaró como pandemia la enfermedad emergente por coronavirus (COVID-19), causada por el virus SARS-CoV-2 (1). El 8 de abril de 2020, las cifras de la pandemia de COVID-19 incluían 1'356.780 casos confirmados, 79.385 muertes y 212 países, áreas o territorios afectados (2).

Los efectos antivirales de la cloroquina fueron descritos en la década de 1960, aunque el interés por ellos se ha renovado desde el 2006 (3). En la epidemia de SARS del 2003 (4), varios investigadores exploraron la efectividad de diferentes moléculas contra el coronavirus SARS-CoV. En el 2004, una concentración efectiva $\left(\mathrm{EC}_{50}\right)$ de $8 \mu \mathrm{M}$ de cloroquina, antes o después de la exposición de las células al virus, demostró eficacia in vitro (5). Según algunos autores, estos hallazgos terminaron siendo olvidados por razones que no están claras $(6,7)$.

La cloroquina es un inhibidor potente de la mayoría de coronavirus, incluido el SARS-CoV (8) y el MERS-CoV (9). Se ha demostrado que el medicamento alcaliniza los endosomas celulares, lo cual entorpece algunos pasos de la replicación viral que requieren un $\mathrm{pH}$ bajo, como la fusión virus-endosoma y el despojo de la cápside vírica (10). Algunos autores han propuesto que la cloroquina también interfiere con la glucosilación de los receptores celulares, como el receptor de la enzima convertidora de angiotensina 2 del SARS-CoV. Además, la cloroquina tiene un efecto inmunomodulador favorable para la eliminación viral (11), al reducir la producción de citocinas proinflamatorias y activar los linfocitos T CD8+ anti-SARS-CoV-2 (12).

Tanto la cloroquina como la hidroxicloroquina podrían ayudar a controlar la 'tormenta citoquímica' experimentada por los pacientes con COVID-19 (13). Dado que la administración de corticoides puede ser deletérea en pacientes con COVID-19 críticamente enfermos (14), los efectos inmunomoduladores de la cloroquina y de la hidroxicloroquina representan un valor agregado de estos medicamentos.

El 4 de febrero de 2020, Wang, et al., publicaron un artículo en la revista Cell Research que recibió la atención del mundo científico (11). Los investigadores evaluaron la actividad de la cloroquina frente al nuevo virus SARS-CoV-2, y encontraron que dosis de $1,13 \mu \mathrm{M}\left(\mathrm{CE}_{50}\right)$ y de $6,90 \mu \mathrm{M}\left(\mathrm{CE}_{90}\right)$ del medicamento prevenían la replicación in vitro del virus en células Vero E6. Según los autores, tales concentraciones pueden alcanzarse fácilmente en seres humanos con la dosis estándar debido a la penetración favorable del fármaco en los tejidos, incluido el pulmón. Como resultado, la cloroquina es hoy la molécula más usada a nivel internacional para el tratamiento de la COVID-19 (12). El 6 de abril de 2020, la cloroquina y la hidroxicloroquina fueron autorizadas por el Ministerio de Salud y Protección Social de Colombia para el tratamiento de esta enfermedad (15).

En un comentario publicado el 5 de marzo de 2020, Touret, et al., reportaron 16 registros de ensayos clínicos que exploraban la efectividad y la seguridad de la cloroquina o la hidroxicloroquina en pacientes con COVID-19 en China (16). Sin embargo, no se sabe si los resultados de alguno de estos estudios ya están publicados, y tampoco se conocen la efectividad y la seguridad del uso de la cloroquina y de sus derivados en pacientes con COVID-19.

La diseminación oportuna de la información científica es clave en tiempos de pandemia. Es necesaria para orientar a los epidemiólogos, a los profesionales de la salud que atienden a los pacientes infectados, a los 
investigadores que exploran las tendencias de la enfermedad y los efectos de las intervenciones en salud e, incluso, para reducir el pánico público (17). En este sentido, es urgente contar con una revisión sistemática que explore la información científica sobre la efectividad y la seguridad de la cloroquina y sus derivados.

La presente revisión sistemática exploratoria aspira a comunicar al público hispano la información científica disponible hasta el 25 de marzo de 2020 sobre el papel de la cloroquina y sus derivados en el manejo de la COVID-19.

\section{Materiales y métodos}

En la revisión se siguieron los pasos propuestos por Arksey, et al. (18) y Levac (19):

i. determinar la pregunta de investigación,

ii. buscar los documentos relevantes,

iii. seleccionar los estudios,

iv. extraer los datos, y

v. resumir y reportar los resultados.

La revisión respondió a la pregunta: ¿cuál es el estado actual de la literatura científica sobre el uso de la cloroquina o sus derivados en el manejo de pacientes con COVID-19? Para responderla, previamente se desarrolló un protocolo (disponible por pedido a los autores).

\section{Criterios de inclusión y exclusión}

Los criterios de inclusión fueron:

i. publicaciones con datos empíricos (in vitro o in vivo) o publicaciones teóricas (revisiones narrativas, comentarios, cartas al editor);

ii. que la publicación estudiara o discutiera el papel de la cloroquina o de cualquiera de sus derivados (hidroxicloroquina, amodiaquina, imiquimod o primaquina);

iii. que presentara resultados de investigación sobre el COVID-19 o el SARS-CoV-2, y

iv. que estuviera en inglés, español, italiano, francés o portugués.

También se incluyeron otros idiomas cuando el resumen estaba en cualquiera de los idiomas considerados en la revisión.

No se consideró límite de fecha en la búsqueda y la última actualización correspondió al 25 de marzo de 2020.

\section{Estrategia de búsqueda}

La búsqueda incluyó las bases de datos PubMed, Embase a través de Ovid, y Lilacs. Usamos operadores booleanos y palabras clave de acuerdo con cada sistema de datos. El algoritmo de búsqueda está disponible en el archivo suplementario 1. Además, se incluyeron las referencias citadas en los documentos si cumplían con los criterios de inclusión y no se habían detectado previamente, así como dos referencias adicionales publicadas después del 25 de marzo sugeridas por expertos en el tema.

Se buscaron los registros de estudios en 15 bases de datos de la Plataforma de Registros Internacionales de Ensayos Clínicos de la OMS (20): Chinese Clinical Trial Registry, U.S. National Library of Medicine, International 
Standard Randomised Controlled Trial Number (ISRCTN), EU Clinical Trials Register (EU-CTR), Japan Primary Registries Network (JPRN), Australian New Zealand Clinical Trials Registry (ANZCTR), Brazilian Clinical Trials Registry (ReBec), Clinical Research Information Service (CRiS), Republic of Korea, Clinical Trials Registry, India (CTRI), Cuban Public Registry of Clinical Trials (RPCEC), German Clinical Trials Register (DRKS), Iranian Registry of Clinical Trials (IRCT), The Netherlands National Trial Register (NTR), Pan African Clinical Trial Registry (PACTR), y Peruvian Clinical Trial Registry (REPEC). Estos registros cumplen con los criterios específicos de contenido, calidad y validez, accesibilidad y capacidad técnica y administrativa (20).

\section{Selección de estudios y extracción de datos}

Usando la aplicación web de acceso gratuito para la gestión de revisiones sistemáticas Rayyan (21), se revisaron y escogieron independientemente los títulos y los resúmenes de las publicaciones candidatas. Los investigadores resolvieron discrepancias mediante discusión y consenso. Posteriormente, se eliminaron los duplicados y se obtuvieron los documentos seleccionados en texto completo a través de la librería de la Universidad McGill (Montreal QC, Canadá).

El siguiente paso fue la extracción de datos. Se usó Microsoft Excel para crear dos formatos basados en las variables que responderían a la pregunta de investigación: un formato para los registros de ensayos clínicos y otro para los documentos restantes. En este paso se hicieron reuniones regulares para discutir y ajustar los formatos.

Se usó el método descriptivo-analítico sugerido por Arksey, et al. (18), para recolectar la información estándar de cada documento. Primero, se calibró el formato usando el $5 \%$ de los documentos para determinar si el método de extracción de datos era adecuado. A continuación, se extrajo la información de todos los documentos restantes.

Se extrajo la siguiente información cuando estaba disponible: autores, título del documento, tipo de documento (comentario, carta al editor, estudio in vitro, estudio clínico), objetivo, idioma, fecha de publicación, revista, país de los autores, y hallazgos principales. En el caso de los registros de ensayos clínicos, se recolectó la siguiente información: código de identificación, estado de reclutamiento, diseño del ensayo, país, tamaño de muestra, intervención, control, medida primaria de resultado, fecha de inicio o de registro, fecha anticipada de terminación y fuente.

\section{Síntesis y presentación de los resultados}

Los resultados de la revisión se presentan siguiendo las categorías propuestas por Grudniewicz, et al. (22):

i. un resumen de las características y la distribución de las publicaciones incluidas, $y$

ii. una síntesis narrativa de los resultados.

En este artículo se empleó la extensión de la declaración PRISMA para reportar revisiones sistemáticas exploratorias (PRISMA-ScR) (23). La lista de chequeo diligenciada está disponible en el archivo suplementario 2. El presente estudio no requirió aprobación ética. 


\section{Resultados}

Se incluyeron 19 documentos y 24 registros de ensayos clínicos ( $n=43)$ (figura 1 y archivo suplementario 3 ).

\section{Ensayos clínicos de exploración de la efectividad y la seguridad de la cloroquina}

Se encontró que el $66 \%$ (16/24) de los ensayos clínicos están registrados en China, dos en el Reino Unido y uno en Estados Unidos, México, Noruega, Corea del Sur, Tailandia y Japón cada uno. Sumando todos los ensayos clínicos, la muestra total es de 18.059 participantes. El ensayo más grande se propone explorar el papel profiláctico de la cloroquina en 10.000 profesionales de la salud a cargo de pacientes con COVID-19 en Inglaterra. Las características de estos ensayos clínicos se describen en el cuadro 1.

El $83 \%$ (20/24) de los ensayos fue controlado y aleatorizado, en tanto que dos $(8 \%)$ son ensayos controlados no aleatorizados y otros dos intervenciones no controladas. Nueve ensayos evalúan la cloroquina únicamente, ocho exclusivamente la hidroxicloroquina y el resto añade un segundo medicamento a cualquiera de estos dos.

En el caso del grupo de control, en el $54 \%$ (12/22) de los estudios controlados se emplea el manejo convencional para la COVID-19, tres usan placebo, tres, medicamentos (arbidol o lopinavir-ritonavir), y en el resto de los ensayos esta información no se determinó. Los ensayos usarán diferentes medidas primarias de resultados, como carga y conversión viral, duración de la enfermedad, mortalidad, síntomas respiratorios y fiebre, y marcadores sanguíneos como la proteína C reactiva. Tres de ellos (NCT04308668, NCT04307693 y NCT04261517) ya están reclutando participantes (23 de marzo de 2020), aunque esta información no está disponible en el Chinese Clinical Trial Registry, por lo que el número es en realidad mayor.

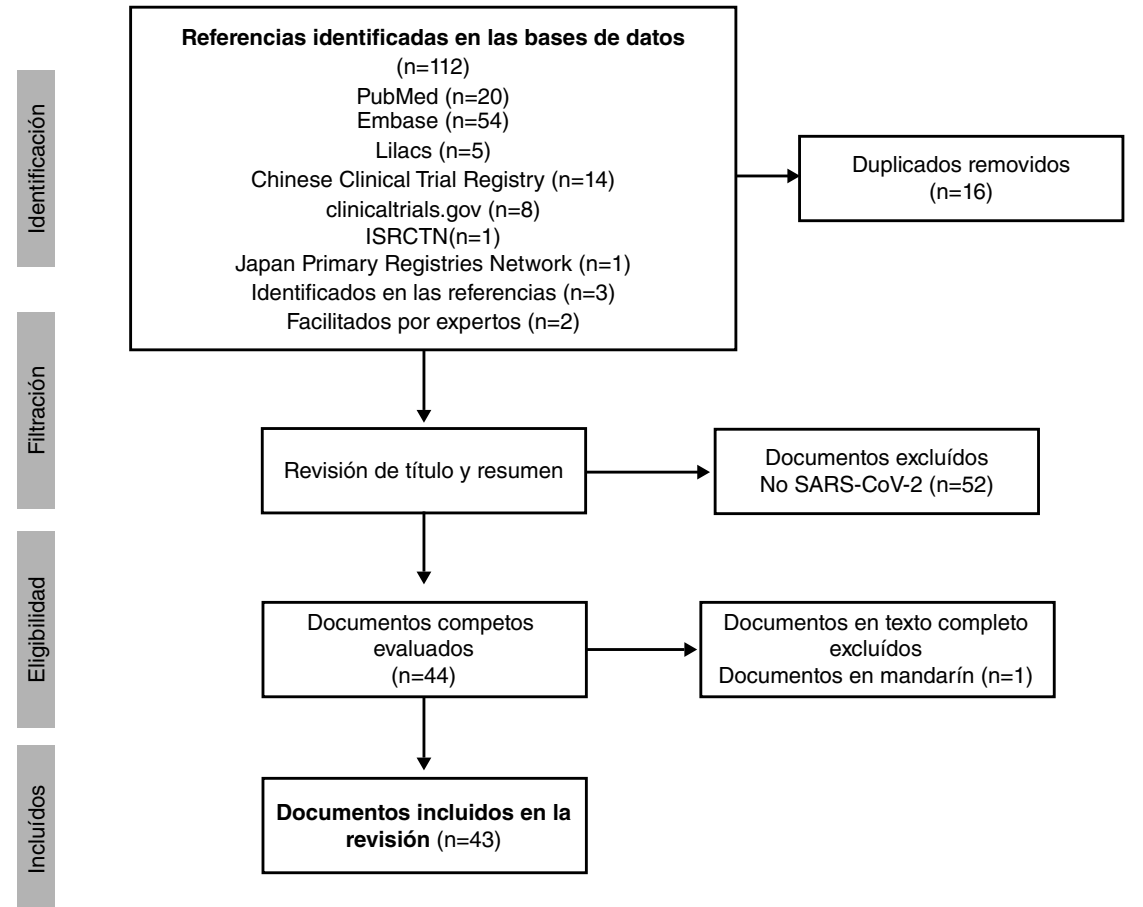

Figura 1. Diagrama PRISMA de la revisión 
Cuadro 1. Características de los ensayos clínicos que exploran la efectividad y la seguridad de la cloroquina o sus derivados en pacientes con COVID-19

\begin{tabular}{|c|c|c|c|c|c|c|c|c|}
\hline ID & Diseño del ensayo & País & $\begin{array}{l}\text { Tamaño de } \\
\text { la muestra }\end{array}$ & Intervención & Control ${ }^{\dagger}$ & $\begin{array}{l}\text { Medida primaria de } \\
\text { resultado }\end{array}$ & $\begin{array}{c}\text { Fecha de inicio } \\
\text { o registro }\end{array}$ & $\begin{array}{l}\text { Fecha esperada } \\
\text { de finalización }\end{array}$ \\
\hline NCT04303507 & $\begin{array}{l}\text { Ensayo controlado } \\
\text { aleatorizado paralelo } \\
\text { doble ciego }^{\text {a }}\end{array}$ & Inglaterra & 10.000 & $\begin{array}{l}\text { Dosis de carga de } 10 \mathrm{mg} / \\
\mathrm{kg} \text { de cloroquina seguida } \\
\text { por } 150 \mathrm{mg} \text { al día por } 3 \\
\text { meses }\end{array}$ & Placebo & $\begin{array}{l}\text { Número de infecciones } \\
\text { sintomáticas COVID-19 }\end{array}$ & Mayo, 2020 & Mayo, 2022 \\
\hline ISRCTN86534580 & $\begin{array}{l}\text { Ensayo controlado } \\
\text { aleatorizado } \\
\text { pragmático basado } \\
\text { en la comunidad }^{\text {a }}\end{array}$ & $\begin{array}{l}\text { Reino } \\
\text { Unido }\end{array}$ & 3.000 & $\begin{array}{l}200 \text { mg sulfato de } \\
\text { hidroxicloroquina dos } \\
\text { al día por } 7 \text { días más } \\
\text { tratamiento usual }\end{array}$ & $\begin{array}{l}\text { Tratamiento } \\
\text { usual }\end{array}$ & $\begin{array}{l}\text { Necesidad de } \\
\text { hospitalización por } \\
\text { COVID-19 }\end{array}$ & $\begin{array}{l}12 \text { de marzo, } \\
2020\end{array}$ & 24 de marzo, 2020 \\
\hline NCT04308668 & $\begin{array}{l}\text { Ensayo controlado } \\
\text { aleatorizado paralelo } \\
\text { de múltiples brazos }^{\text {b }}\end{array}$ & $\begin{array}{l}\text { Estados } \\
\text { Unidos }\end{array}$ & 1.500 & $\begin{array}{l}800 \mathrm{mg} \text { de } \\
\text { hidroxicloroquina } \\
\text { seguidos por } 600 \mathrm{mg} \text { tras } \\
6-8 \text { horas, seguidos por } \\
600 \mathrm{mg} \text { al día por } 6 \text { días }\end{array}$ & $\begin{array}{l}\text { Placebo } \\
\text { siguiendo el } \\
\text { régimen de } \\
\text { intervención }\end{array}$ & $\begin{array}{l}\text { Incidencia de } \\
\text { COVID-19 y escala } \\
\text { de gravedad de la } \\
\text { infección }\end{array}$ & $\begin{array}{l}17 \text { de marzo, } \\
2020\end{array}$ & Mayo, 2021 \\
\hline NCT04286503 & $\begin{array}{l}\text { Ensayo controlado } \\
\text { aleatorizado paralelo }^{\text {a }}\end{array}$ & China & 520 & $\begin{array}{l}\text { Fosfato de cloroquina o } \\
\text { lopinavir/ritonavir }\end{array}$ & $\begin{array}{l}\text { Tratamiento } \\
\text { básico }\end{array}$ & $\begin{array}{l}\text { Fiebre; resolución de la } \\
\text { inflamación pulmonar; } \\
\text { conversión negativa de } \\
\text { SARS-CoV-19 en frotis } \\
\text { de garganta }\end{array}$ & $\begin{array}{l}23 \text { de febrero, } \\
2020\end{array}$ & 28 de febrero, 2021 \\
\hline NCT04315896 & $\begin{array}{l}\text { Ensayo controlado } \\
\text { aleatorizado de dos } \\
\text { brazos, doble ciego, } \\
\text { paralelo a }^{\text {a }}\end{array}$ & México & 500 & $\begin{array}{l}200 \text { mg de } \\
\text { hidroxicloroquina cada } 12 \\
\text { horas por } 10 \text { días }\end{array}$ & $\begin{array}{l}\text { Placebo } \\
\text { siguiendo el } \\
\text { régimen de } \\
\text { intervención }\end{array}$ & $\begin{array}{l}\text { Mortalidad hospitalaria } \\
\text { por todas las causas }\end{array}$ & $\begin{array}{l}23 \text { de marzo, } \\
2020\end{array}$ & 22 de marzo, 2021 \\
\hline ChiCTR2000029868 & $\begin{array}{l}\text { Ensayo controlado } \\
\text { aleatorizado } \\
\text { multicéntrico abierto }\end{array}$ & China & 360 & $\begin{array}{l}\text { Sulfato de } \\
\text { hidroxicloroquina oral }\end{array}$ & $\begin{array}{l}\text { Tratamiento } \\
\text { convencional } \\
\text { para COVID-19 }\end{array}$ & $\begin{array}{l}\text { Test de ácido nucleico } \\
\text { viral }\end{array}$ & $\begin{array}{l}15 \text { de febrero, } \\
2020\end{array}$ & No disponible \\
\hline ChiCTR2000029803 & $\begin{array}{l}\text { Ensayo controlado } \\
\text { aleatorizado abierto }\end{array}$ & China & 320 & Hidroxicloroquina & $\begin{array}{l}\text { Clorhidrato de } \\
\text { arbidol }\end{array}$ & $\begin{array}{l}\text { Progresión de } \\
\text { sospechoso a infectado } \\
\text { por SARS-CoV-2 }\end{array}$ & $\begin{array}{l}14 \text { de febrero, } \\
2020\end{array}$ & No disponible \\
\hline ChiCTR2000029559 & $\begin{array}{l}\text { Ensayo controlado } \\
\text { aleatorizado paralelo }\end{array}$ & China & 300 & Hidroxicloroquina & $\begin{array}{l}\text { Comprimido de } \\
\text { almidón }\end{array}$ & $\begin{array}{l}\text { Conversión viral } \\
\text { negativa; tiempo } \\
\text { de recuperación de } \\
\text { células T }\end{array}$ & $\begin{array}{l}04 \text { de febrero, } \\
2020\end{array}$ & No disponible \\
\hline ChiCTR2000029609 & $\begin{array}{l}\text { Ensayo controlado } \\
\text { aleatorizado } \\
\text { multicéntrico abierto }\end{array}$ & China & 205 & $\begin{array}{l}\text { Brazo 1: fosfato de } \\
\text { cloroquina } \\
\text { Brazo 2: lopinavir/ritonavir }\end{array}$ & $\begin{array}{l}\text { Cloroquina más } \\
\text { lopinavir/ritonavir }\end{array}$ & $\begin{array}{l}\text { Conversión viral } \\
\text { negativa }\end{array}$ & $\begin{array}{l}06 \text { de febrero, } \\
2020\end{array}$ & No disponible \\
\hline $\begin{array}{l}\text { ClinicalTrials.gov: } \\
\text { NCT04316377 } \\
\text { EudraCT Number: } \\
\text { 2020-001010-38 }\end{array}$ & $\begin{array}{l}\text { Ensayo controlado } \\
\text { aleatorizado de dos } \\
\text { brazos pragmático }^{\text {paralelo }^{\text {a }}}\end{array}$ & Noruega & 202 & $\begin{array}{l}400 \text { mg hidroxicloroquina } \\
\text { dos veces al día por } 7 \\
\text { días más tratamiento } \\
\text { estándar }\end{array}$ & $\begin{array}{l}\text { Tratamiento } \\
\text { estándar }\end{array}$ & $\begin{array}{l}\text { Tasa de disminución en } \\
\text { la carga viral de SARS- } \\
\text { CoV-2 }\end{array}$ & $\begin{array}{l}23 \text { de marzo, } \\
2020\end{array}$ & $\begin{array}{c}03 \text { de marzo de } \\
2025\end{array}$ \\
\hline NCT04307693 & $\begin{array}{l}\text { Ensayo controlado } \\
\text { aleatorizado paralelo } \\
\text { multicéntrico }^{b}\end{array}$ & $\begin{array}{l}\text { Corea del } \\
\text { Sur }\end{array}$ & 150 & $\begin{array}{l}400 \mathrm{mg} \text { de } \\
\text { hidroxicloroquina cada } 12 \\
\text { horas por } 7-10 \text { días }\end{array}$ & $\begin{array}{l}\text { Sin } \\
\text { hidroxicloroquina }\end{array}$ & $\begin{array}{l}\text { Carga viral SARS- } \\
\text { CoV-19 }\end{array}$ & $\begin{array}{l}11 \text { de marzo, } \\
2020\end{array}$ & Mayo de 2020 \\
\hline ChiCTR2000030987 & $\begin{array}{l}\text { Ensayo controlado } \\
\text { aleatorizado paralelo }\end{array}$ & China & 150 & $\begin{array}{l}\text { Favapivir más fosfato de } \\
\text { cloroquina }\end{array}$ & Placebo & $\begin{array}{l}\text { Mejoría de síntomas } \\
\text { respiratorios }\end{array}$ & $\begin{array}{l}20 \text { de marzo, } \\
2020^{\star}\end{array}$ & No disponible \\
\hline ChiCTR2000029741 & $\begin{array}{l}\text { Ensayo controlado } \\
\text { aleatorizado } \\
\text { multicéntrico, abierto }\end{array}$ & China & 112 & Fosfato de cloroquina & $\begin{array}{l}\text { Lopinavir / } \\
\text { ritonavir }\end{array}$ & $\begin{array}{l}\text { Duración de la } \\
\text { enfermedad; } \\
\text { mortalidad por todas } \\
\text { las causas; valores } \\
\text { del hemograma, } \\
\text { marcadores de } \\
\text { inflamación, } \\
\text { indicadores de } \\
\text { coagulación, ácido } \\
\text { nucleico viral }\end{array}$ & $\begin{array}{l}11 \text { de febrero, } \\
2020^{\star}\end{array}$ & No disponible \\
\hline ChiCTR2000030054 & $\begin{array}{l}\text { Ensayo controlado } \\
\text { aleatorizado abierto }\end{array}$ & China & 100 & $\begin{array}{l}\text { Brazo 1: fosfato de } \\
\text { cloroquina } \\
\text { Brazo 2: sulfato de } \\
\text { hidroxicloroquina }\end{array}$ & $\begin{array}{l}\text { Tratamiento } \\
\text { recomendado } \\
\text { para COVID-19 }\end{array}$ & $\begin{array}{l}\text { Tiempo de mejoría } \\
\text { clínica }\end{array}$ & $\begin{array}{l}22 \text { de febrero, } \\
2020^{\star}\end{array}$ & No disponible \\
\hline ChiCTR2000029992 & $\begin{array}{l}\text { Ensayo controlado } \\
\text { aleatorizado abierto }\end{array}$ & China & 100 & $\begin{array}{l}\text { Brazo 1: fosfato de } \\
\text { cloroquina } \\
\text { Brazo 2: sulfato de } \\
\text { hidroxicloroquina }\end{array}$ & $\begin{array}{l}\text { Tratamiento } \\
\text { recomendado } \\
\text { para COVID-19 }\end{array}$ & $\begin{array}{l}\text { Tiempo de mejoría } \\
\text { clínica; cambios en la } \\
\text { carga viral en muestras } \\
\text { de vías respiratorias } \\
\text { superior e inferior }\end{array}$ & $\begin{array}{l}18 \text { de febrero, } \\
2020^{\star}\end{array}$ & No disponible \\
\hline ChiCTR2000029939 & $\begin{array}{l}\text { Ensayo controlado } \\
\text { aleatorizado }\end{array}$ & China & 100 & $\begin{array}{l}\text { Fosfato de cloroquina más } \\
\text { tratamiento convencional } \\
\text { para COVID-19 }\end{array}$ & $\begin{array}{l}\text { Tratamiento } \\
\text { convencional } \\
\text { para COVID-19 }\end{array}$ & $\begin{array}{l}\text { Duración de } \\
\text { hospitalización }\end{array}$ & $\begin{array}{l}16 \text { de febrero, } \\
2020^{\star}\end{array}$ & No disponible \\
\hline
\end{tabular}




\begin{tabular}{|c|c|c|c|c|c|c|c|c|}
\hline ChiCTR2000029935 & $\begin{array}{l}\text { Intervención de un } \\
\text { solo brazo }\end{array}$ & China & 100 & $\begin{array}{l}\text { Fosfato de cloroquina más } \\
\text { tratamiento convencional } \\
\text { para COVID-19 }\end{array}$ & $\begin{array}{l}\text { Sin grupo de } \\
\text { control }\end{array}$ & $\begin{array}{l}\text { Duración de } \\
\text { hospitalización }\end{array}$ & $\begin{array}{l}16 \text { de febrero, } \\
2020^{*}\end{array}$ & No disponible \\
\hline NCT04303299 & $\begin{array}{l}\text { Ensayo controlado } \\
\text { aleatorizado paralelo } \\
\text { de múltiples brazos }^{\text {a }}\end{array}$ & Tailandia & 80 & $\begin{array}{l}\text { Tres brazos usarán } \\
\text { cloroquina más varios } \\
\text { antivirales (oseltamivir, } \\
\text { darunavir, ritonavir) }\end{array}$ & $\begin{array}{l}\text { Cuarentena } \\
\text { convencional }\end{array}$ & $\begin{array}{l}\text { Tiempo de erradicación } \\
\text { del SARS-CoV-2 }\end{array}$ & $\begin{array}{l}15 \text { de marzo, } \\
2020\end{array}$ & $\begin{array}{c}30 \text { de noviembre, } \\
2020\end{array}$ \\
\hline ChiCTR2000030718 & $\begin{array}{l}\text { Ensayo controlado } \\
\text { aleatorizado paralelo }\end{array}$ & China & 80 & Fosfato de cloroquina & Nada & $\begin{array}{l}\text { Tiempo de mejoría } \\
\text { clínica }\end{array}$ & $\begin{array}{l}11 \text { de marzo, } \\
2020^{\star}\end{array}$ & No disponible \\
\hline ChiCTR2000029988 & Ensayo controlado & China & 80 & Fosfato de cloroquina & Nada & $\begin{array}{l}\text { Tiempo de mejoría } \\
\text { clínica }\end{array}$ & $\begin{array}{l}18 \text { de febrero, } \\
2020^{\star}\end{array}$ & No disponible \\
\hline jRCTs031190227 & $\begin{array}{l}\text { Intervención } \\
\text { multicéntrica no } \\
\text { controlada }^{a}\end{array}$ & Japón & 50 & $\begin{array}{l}\text { Lopinavir, ritonavir e } \\
\text { hidroxicloroquina con o } \\
\text { sin oseltamivir }\end{array}$ & No mencionado & $\begin{array}{l}\text { Proteína C reactiva } \\
\text { antes y después del } \\
\text { tratamiento }\end{array}$ & $\begin{array}{l}27 \text { de febrero, } \\
2020\end{array}$ & No disponible \\
\hline NCT04261517 & $\begin{array}{l}\text { Ensayo controlado } \\
\text { aleatorizado paralelo }^{\mathrm{b}}\end{array}$ & China & 30 & $\begin{array}{l}400 \mathrm{mg} \text { de } \\
\text { hidroxicloroquina al } \\
\text { día por } 5 \text { días más } \\
\text { tratamiento convencional }\end{array}$ & $\begin{array}{l}\text { Tratamiento } \\
\text { convencional }\end{array}$ & $\begin{array}{l}\text { Tasa de eliminación } \\
\text { viral en esputo, } \\
\text { frotis de garganta o } \\
\text { secreciones de vía } \\
\text { respiratoria inferior; } \\
\text { tasa de mortalidad de } \\
\text { participantes }\end{array}$ & $\begin{array}{c}06 \text { de febrero, } \\
2020\end{array}$ & $\begin{array}{l}31 \text { de diciembre, } \\
2020\end{array}$ \\
\hline ChiCTR2000029542 & $\begin{array}{l}\text { Ensayo controlado } \\
\text { no aleatorizado }\end{array}$ & China & 20 & Cloroquina & $\begin{array}{l}\text { Manejo } \\
\text { convencional }\end{array}$ & $\begin{array}{l}\text { Conversión viral } \\
\text { negativa; mortalidad } \\
\text { por todas las causas a } \\
30 \text { días }\end{array}$ & $\begin{array}{l}03 \text { de febrero, } \\
2020^{\star}\end{array}$ & No disponible \\
\hline ChiCTR2000029975 & $\begin{array}{l}\text { Intervención de un } \\
\text { solo brazo }\end{array}$ & China & 10 & Fosfato de cloroquina & $\begin{array}{l}\text { Sin grupo de } \\
\text { control }\end{array}$ & $\begin{array}{l}\text { Tiempo de conversión } \\
\text { viral negativa; } \\
\text { mortalidad por todas } \\
\text { las causas }\end{array}$ & $\begin{array}{l}18 \text { de febrero, } \\
2020^{\star}\end{array}$ & No disponible \\
\hline
\end{tabular}

a No iniciado; ${ }^{b}$ Iniciado; ${ }^{\dagger}$ Los detalles del manejo convencional no se describen en los registros; * Fecha de registro

Dos ensayos (NCT04303299 y ISRCTN86534580) planean terminar en el 2020, tres en el 2021, tres en el 2022, uno en el 2020 y el último en el 2025. En el resto de los ensayos la fecha es incierta.

\section{Síntesis de los hallazgos de las publicaciones incluidas en la revisión}

Nueve de los 19 documentos incluidos son comentarios, cartas o editoriales, tres son estudios in vitro, dos son revisiones narrativas sobre la farmacología de la cloroquina y la hidroxicloroquina, dos son guías de práctica clínica, uno es una revisión sistemática y otro, un consenso de expertos (archivo suplementario 3). Solo se encontró un ensayo controlado. Dieciséis documentos están en inglés y los otros tres en mandarín, italiano y holandés cada uno. Los documentos en mandarín y holandés tienen resumen en inglés en concordancia con los criterios de inclusión. Ocho documentos son de China, cinco de Francia, dos de Italia, dos de Estados Unidos, uno de Irán y el otro de Holanda. Los hallazgos principales de cada uno de estos documentos se describen en el cuadro 2.

El 4 de febrero de 2020, Wang, et al., publicaron en la revista Cell Research el primer estudio sobre la actividad de la cloroquina en el SARSCoV-2 (11). Reportaron que esta previene la replicación in vitro del virus en células Vero E6 y animaron a la comunidad científica a investigar la efectividad del medicamento en pacientes con COVID-19.

En una publicación del 19 de febrero de 2020, Gao, et al., reportaron que los "resultados de más de 100 pacientes demostraron que el fosfato de cloroquina es superior al tratamiento de control al inhibir la exacerbación de la neumonía, mejorar los hallazgos en las imágenes diagnósticas pulmonares (tomografía axial computarizada), promover la conversión negativa del virus, y acortar la duración de la enfermedad." (24). No se reportaron reacciones adversas graves al medicamento. 
Cuadro 2. Características de las publicaciones incluidas en la revisión

\begin{tabular}{|c|c|c|c|c|c|c|}
\hline Autores & $\begin{array}{l}\text { Tipo de } \\
\text { documento }\end{array}$ & Objetivo & $\begin{array}{l}\text { Fecha de publicación } \\
\text { (año: 2020) }\end{array}$ & Revista & $\begin{array}{l}\text { País de los } \\
\text { autores }\end{array}$ & Hallazgo principal / contribución \\
\hline $\begin{array}{l}\text { Wang M, Cao R, Zhang L, } \\
\text { Yang X, Liu J, Xu M, et al. }\end{array}$ & $\begin{array}{l}\text { Carta al editor - } \\
\text { Estudio in vitro a }\end{array}$ & $\begin{array}{l}\text { Evaluar la eficiencia antiviral } \\
\text { de cinco medicamentos } \\
\text { aprobados por la FDA y } \\
\text { dos antivirales de amplio } \\
\text { espectro en el COVID-19 }\end{array}$ & 04 de febrero & Cell Research & China & $\begin{array}{l}\text { Primer estudio en reportar actividad } \\
\text { de la cloroquina in vitro frente al } \\
\text { SARS-CoV-2. }\end{array}$ \\
\hline $\begin{array}{l}\text { Colson P, Rolain JM, } \\
\text { Raoult D }\end{array}$ & $\begin{array}{l}\text { Comentario } \\
\text { - sección Hot } \\
\text { Topic }^{\text {a }}\end{array}$ & NA & 15 de febrero & $\begin{array}{l}\text { International } \\
\text { Journal of } \\
\text { Antimicrobial } \\
\text { Agents }\end{array}$ & Francia & $\begin{array}{l}\text { Los investigadores, expertos en } \\
\text { hidroxicloroquina en infecciones } \\
\text { intracelulares, resaltan la importancia } \\
\text { del hallazgo de Wang, et al. Plantean } \\
\text { potencial uso profiláctico y terapéutico } \\
\text { del medicamento en COVID-19. }\end{array}$ \\
\hline $\begin{array}{l}\text { Multicenter collaboration } \\
\text { group of Department of } \\
\text { Science and Technology } \\
\text { of Guangdong Province } \\
\text { for chloroquine in the } \\
\text { treatment of novel } \\
\text { coronavirus pneumonia }\end{array}$ & $\begin{array}{l}\text { Consenso de } \\
\text { expertos }^{b *}\end{array}$ & $\begin{array}{l}\text { Guiar y regular el uso de } \\
\text { cloroquina en pacientes con } \\
\text { neumonía por SARS-CoV-2 }\end{array}$ & 20 de febrero & $\begin{array}{l}\text { Zhonghua Jie } \\
\text { He He Hu Xi Za } \\
\text { Zhi (Chinese } \\
\text { Journal of } \\
\text { Tuberculosis } \\
\text { and Respiratory } \\
\text { Diseases) }\end{array}$ & China * & $\begin{array}{l}\text { Recomendó el tratamiento de } \\
\text { COVID-19 con fosfato de cloroquina, } \\
500 \mathrm{mg} \text {, dos veces al día, durante } 10 \\
\text { días para pacientes diagnosticados } \\
\text { con neumonía por SARS-CoV-2 } \\
\text { leve y moderada a grave, sin } \\
\text { contraindicación para la cloroquina. }\end{array}$ \\
\hline Dong L, Hu S, Gao J & Comentario $^{\text {a }}$ & NA & 29 de febrero & $\begin{array}{l}\text { Drug } \\
\text { Discoveries \& } \\
\text { Therapeutics }\end{array}$ & China & $\begin{array}{l}\text { Comenta que el fosfato de cloroquina } \\
\text { ha sido incluido en la sexta edición } \\
\text { de las guías para la prevención, } \\
\text { diagnóstico, y tratamiento de la } \\
\text { neumonía por el nuevo coronavirus } \\
\text { de la Comisión Nacional de } \\
\text { Salud del gobierno chino. Discute } \\
\text { otros medicamentos con efectos } \\
\text { potenciales sobre el SARS CoV-2 } \\
\text { como arbidol, remdesivir, y favipiravir. }\end{array}$ \\
\hline Gao J, Tian Z, Yang X & Comentario $^{a}$ & NA & 29 de febrero & $\begin{array}{l}\text { BioScience } \\
\text { Trends }\end{array}$ & China & $\begin{array}{l}\text { Reporta que los resultados en } 100 \\
\text { pacientes indican que el fosfato de } \\
\text { cloroquina es superior al tratamiento } \\
\text { de control en pacientes con } \\
\text { neumonía por SARS-CoV-2. }\end{array}$ \\
\hline Song $\mathrm{P}$, Karako $\mathrm{T}$ & Editorial a & NA & 29 de febrero & $\begin{array}{l}\text { BioScience } \\
\text { Trends }\end{array}$ & China & $\begin{array}{l}\text { Resalta el papel potencial de la } \\
\text { cloroquina en el tratamiento de } \\
\text { COVID-19. }\end{array}$ \\
\hline $\begin{array}{l}\text { Colson P, Rolain JM, } \\
\text { Lagier JC, Brouqui P, } \\
\text { Raoult D }\end{array}$ & $\begin{array}{l}\text { Comentario } \\
\text { - sección Hot } \\
\text { Topic }^{\text {a }}\end{array}$ & NA & 04 de marzo & $\begin{array}{l}\text { International } \\
\text { Journal of } \\
\text { Antimicrobial } \\
\text { Agents }\end{array}$ & Francia & $\begin{array}{l}\text { Resalta hallazgos de Wang, et } \\
\text { al. y Gao, et al., y sugiere que la } \\
\text { hidroxicloroquina podría ser también } \\
\text { efectiva contra el SARS-CoV-2. } \\
\text { Resume estudios sobre la actividad } \\
\text { de la cloroquina o la hidroxicloroquina } \\
\text { en coronavirus (no SARS-CoV-2). }\end{array}$ \\
\hline Touret F, Lamballerie $\mathrm{X}$ & Comentario $^{a}$ & NA & 05 de marzo & $\begin{array}{l}\text { Antiviral } \\
\text { Research }\end{array}$ & Francia & $\begin{array}{l}\text { Llama la atención sobre la necesidad } \\
\text { de interpretar los hallazgos de Wang, } \\
\text { et al. y Gao, et al. con precaución. }\end{array}$ \\
\hline $\begin{array}{l}\text { Yao X, Ye F, Zhang M, Cui } \\
\text { C, Huang B, Niu P, et al. }\end{array}$ & Estudio in vitro a & $\begin{array}{l}\text { Explorar la actividad } \\
\text { farmacológica de la cloroquina } \\
\text { y la hidroxicloroquina en } \\
\text { células Vero infectadas con } \\
\text { SARS-CoV-2 }\end{array}$ & 09 de marzo & $\begin{array}{l}\text { Clinical } \\
\text { Infectious } \\
\text { Diseases }\end{array}$ & China & $\begin{array}{l}\text { Encontraron que la hidroxicloroquina } \\
\text { es más potente que la cloroquina in } \\
\text { vitro. }\end{array}$ \\
\hline $\begin{array}{l}\text { Cortegiani A, Ingoglia G, } \\
\text { Ippolito M, Giarratano A, } \\
\text { Einav S }\end{array}$ & $\begin{array}{l}\text { Revisión } \\
\text { sistemática a }\end{array}$ & $\begin{array}{l}\text { Explorar la evidencia sobre } \\
\text { la efectividad y la seguridad } \\
\text { de la cloroquina para el } \\
\text { tratamiento de COVID-19 }\end{array}$ & 10 de marzo & $\begin{array}{l}\text { Journal of } \\
\text { Critical Care }\end{array}$ & Italia e Israel & $\begin{array}{l}\text { Encontraron } 6 \text { publicaciones y } 23 \\
\text { ensayos clínicos en curso. }\end{array}$ \\
\hline $\begin{array}{l}\text { Devaux CA, Rolain JM, } \\
\text { Colson P, Raoult }\end{array}$ & $\begin{array}{l}\text { Revisión } \\
\text { narrativa }^{\text {a }}\end{array}$ & $\begin{array}{l}\text { Describir los posibles } \\
\text { mecanismos de acción } \\
\text { de la cloroquina sobre la } \\
\text { replicación de SARS-CoV-2 }\end{array}$ & 12 de marzo & $\begin{array}{l}\text { International } \\
\text { Journal of } \\
\text { Antimicrobial } \\
\text { Agents }\end{array}$ & Francia & $\begin{array}{l}\text { Describe en detalle los varios } \\
\text { mecanismos de acción antivirales de } \\
\text { la cloroquina. }\end{array}$ \\
\hline $\begin{array}{l}\text { Sahraei Z, Shabani M, } \\
\text { Shokouhi S, Saffaei A }\end{array}$ & Carta al editor ${ }^{a}$ & NA & 17 de marzo & $\begin{array}{l}\text { International } \\
\text { Journal of } \\
\text { Antimicrobial } \\
\text { Agents }\end{array}$ & Irán & $\begin{array}{l}\text { Comenta que en algunos países } \\
\text { como Irán, la disponibilidad de la } \\
\text { cloroquina es limitada, por lo cual la } \\
\text { hidroxicloroquina es una opción válida. }\end{array}$ \\
\hline $\begin{array}{l}\text { Liu J, Cao R, Xu M, Wang } \\
\text { X, Zhang } \mathrm{H}, \mathrm{Hu} \mathrm{H} \text {, et al. }\end{array}$ & $\begin{array}{l}\text { Carta al editor - } \\
\text { Estudio in vitro a }\end{array}$ & $\begin{array}{l}\text { Evaluar el efecto antiviral } \\
\text { de la hidroxicloroquina } \\
\text { contra el SARS-CoV-2, } \\
\text { en comparación con la } \\
\text { cloroquina in vitro }\end{array}$ & 18 de marzo & Cell Discovery & China & $\begin{array}{l}\text { Reportan un efecto más potente } \\
\text { de la cloroquina, en comparación } \\
\text { con la hidroxicloroquina. Reportan } \\
7 \text { ensayos clínicos en curso que } \\
\text { exploran la efectividad de la } \\
\text { hidroxicloroquina en China. }\end{array}$ \\
\hline
\end{tabular}


Zhou D, Dai SM, Tong Q $\begin{aligned} & \text { Revisión } \\ & \text { narrativa }^{\text {a }}\end{aligned}$
Gautret P, Lagier J, Parola Ensayo

P, Hoang VT, Meddeb L, controlado ${ }^{a}$

Mailhe $M$

Sociedad Italiana de

Enfermedades Infecciosas

y Tropicales (Simit,

acrónimo en italiano)

Grupo de Trabajo

Holandés sobre Política

Antibiótica (SWAB

acrónimo en holandés)

Wilson FP

Hinton DM

\section{Guía de práctica}

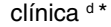

Comentario $^{\text {a }}$

Carta $^{\dagger}$

\section{Evaluar el efecto de la \\ hidroxicloroquina en \\ pacientes infectados con \\ SARS-CoV-2}

Guía de práctica

NA

NA

Revisar la calidad del estudio de Gautret, et al.

Autorizar uso de emergencia de medicamentos en Estados Unidos
20 de marzo $\quad \begin{aligned} & \text { Journal of } \\ & \text { Antimicrobial }\end{aligned}$

Chemotherapy

20 de marzo

International

Journal of

Antimicrobial

Agents

Marzo

Marzo

Marzo, 2026

Marzo, 2028
NA

NA

NA

NA
China

Resalta el potencial rol de la hidroxicloroquina en modular la "tormenta citoquímica". Comenta que es más segura que la cloroquina y que puede ser usada en mujeres embarazadas. También, que es más económica y está disponible en China.

Francia Primer estudio experimental sobre efectividad de la hidroxicloroquina en pacientes con COVID-19

Italia

Recomienda uso de cloroquina o hidroxicloroquina para manejo de pacientes con COVID-19.

Holanda *

Recomienda el uso de cloroquina o hidroxicloroquina para el manejo de pacientes con COVID-19.

Presenta los fallos metodológicos del estudio.

Emitió una Autorización de Uso de Emergencia para el fosfato de cloroquina y el sulfato de hidroxicloroquina en Estados Unidos.

a Inglés; ${ }^{\mathrm{b}}$ Mandarín; ${ }^{\mathrm{c}}$ Italiano; ${ }^{\mathrm{d}}$ Holandés

* Solo se revisaron los resúmenes de estos documentos

† Documentos sugeridos por expertos aparecidos después de marzo 25, 2020

NA: no aplica

En su comentario publicado el 5 de marzo de 2020, Touret, et al., piden mesura frente a los hallazgos de Gao, et al. (16). Comentan que si bien el hallazgo es una noticia excelente dado el bajo costo y la disponibilidad de la cloroquina en todo el mundo, los resultados deben interpretarse con precaución, pues los datos que respaldan las conclusiones del estudio aún no están disponibles. Además, según ellos, el estudio de Gao, et al., se realizó en 10 hospitales diferentes, con protocolos clínicos distintos, diversos grupos de control (placebo, otros antivirales, nada, etc.), y variables heterogéneas.

Posteriormente, el 9 de marzo, Yao, et al., publicaron un estudio in vitro comparando la actividad farmacológica de la cloroquina y la hidroxicloroquina en células Vero 6 infectadas con SARS-CoV-2. Encontraron que la hidroxicloroquina $\left(E C_{50}=0,72 \mu \mathrm{M}\right)$ también es eficaz contra el SARS-CoV-2 y es más potente que la cloroquina $\left(\mathrm{EC}_{50}=5,47 \mu \mathrm{M}\right)$ in vitro (25).

El 18 de marzo de 2020, Liu, et al., publicaron un estudio en el que exploraron el efecto antiviral in vitro de la hidroxicloroquina contra el SARSCoV-2 en comparación con la cloroquina (26). Reportaron valores de $\mathrm{EC}_{50}$ de $2,71,3,81,7,14$ y $7,36 \mu \mathrm{M}$ de cloroquina y de $4,51,4,06,17,31$ y $12,96 \mu \mathrm{M}$ de hidroxicloroquina para diferentes multiplicidades de infección. Estos datos sugieren un efecto más potente de la cloroquina que de la hidroxicloroquina (diferencias estadísticamente significativas). Usando técnicas de inmunofluorescencia, los autores también reportaron que ambos medicamentos bloquean el transporte del SARS-CoV-2 desde los endosomas tempranos hasta los endolisosomas, lo que parece ser un requisito para liberar el genoma vírico a nivel intracelular. Asimismo, observaron cambios morfológicos de los endosomas tempranos en las células expuestas a cualquiera de los dos medicamentos, lo que sugiere un bloqueo de la maduración en las etapas intermedias de la endocitosis que dificultaría el transporte del virión al sitio de liberación intracelular. 
El 20 de marzo de 2020, los investigadores franceses Gautret, et al., publicaron el primer ensayo clínico sobre la efectividad de la hidroxicloroquina en pacientes infectados con SARS-CoV-2 (27). El ensayo no fue aleatorizado, pero sí contó con grupo de control; 26 pacientes recibieron $200 \mathrm{mg}$ del medicamento tres veces al día durante 10 días. Algunos de los pacientes recibieron azitromicina adicional para prevenir la infección bacteriana. Después de seis días de tratamiento encontraron, mediante reacción en cadena de la polimerasa en cultivo de frotis nasofaríngeo, que el $70 \%$ de los pacientes en el grupo de intervención estaba virológicamente curado, comparado con el 12,5\% de los 16 pacientes del grupo de control. Más aún, al día seis de la intervención todos los pacientes que habían recibido una combinación de hidroxicloroquina y azitromicina estaban virológicamente curados, en comparación con el $57 \%$ de los pacientes que habían recibido únicamente hidroxicloroquina y del 12,5 $\%$ de los pacientes del grupo control. Seis pacientes del grupo de intervención no finalizaron el estudio por diferentes razones: tres casos fueron transferidos a la unidad de cuidados intensivos, uno murió y los otros dos interrumpieron su tratamiento. Estos pacientes no fueron incluidos en el análisis final.

Los autores concluyeron que el tratamiento con hidroxicloroquina se asociaba significativamente con una reducción o desaparición de la carga viral en pacientes con COVID-19 y que, además, este efecto era sinérgico con la azitromicina. Los investigadores recomendaron que los pacientes con COVID-19 sean tratados con hidroxicloroquina y azitromicina para curar su infección y para limitar la transmisión del virus a otras personas. Sugirieron que deben hacerse nuevos estudios para determinar si la hidroxicloroquina puede usarse como quimioprofilaxis contra la COVID-19, especialmente en trabajadores de la salud.

Al analizar el estudio de Gautret, et al., Wilson comentó que hubo pérdida diferencial e importante durante el seguimiento a los pacientes en los dos grupos del estudio (28). A diferencia del grupo de intervención, ningún paciente del grupo control murió o fue remitido a la unidad de cuidados intensivos. Según Wilson, si estos seis pacientes hubieran sido incluidos en el análisis, la hidroxicloroquina hubiese estado asociada con un aumento del riesgo de muerte y transferencia a la unidad de cuidados intensivos en los pacientes con COVID-19. El descartar a los seis pacientes mencionados permitió a los autores reportar un aparente impacto positivo de la hidroxicloroquina en su estudio.

El 28 de marzo de 2020, la Food and Drug Administration (FDA) de Estados Unidos emitió una "autorización de uso de emergencia" para el fosfato de cloroquina y el sulfato de hidroxicloroquina. La institución autorizó el uso de estos medicamentos para tratar pacientes adultos o adolescentes con peso de $50 \mathrm{~kg}$ o más hospitalizados con COVID-19, para quienes la participación en ensayos clínicos no estuviera disponible o no fuera posible (29).

\section{Discusión}

Se encontraron 24 registros de ensayos clínicos y 19 documentos sobre el efecto de la cloroquina o la hidroxicloroquina en la COVID-19. La mayoría de los ensayos clínicos son aleatorizados, se desarrollan en China y en total acumulan un tamaño de muestra de más de 18.000 pacientes. Un número similar de ensayos evalúan los efectos de la cloroquina y la hidroxicloroquina, a veces combinadas con un segundo medicamento. La mayoría de los ensayos utiliza el tratamiento convencional en el grupo de control, aunque los registros no describen los componentes del tratamiento convencional y emplean diferentes tipos de variables. 
La mayoría de los documentos incluidos son teóricos y solo cuatro son estudios empíricos. De estos, se encontró solamente un ensayo controlado no aleatorizado en humanos. Los datos de los estudios empíricos sugieren que la cloroquina y la hidroxicloroquina son efectivas para controlar la replicación del virus SARS-CoV-2 y, tal vez, para mejorar el curso de la COVID-19. Sin embargo, las conclusiones del único ensayo clínico disponible no son concluyentes (27) debido al reducido tamaño de la muestra y al deficiente diseño del estudio (28). Tampoco se cuenta con los datos del ensayo clínico de Gao, et al., en 100 pacientes (24).

Los autores de los documentos incluidos en esta revisión comentan que la cloroquina y la hidroxicloroquina son medicamentos generalmente seguros, y que los efectos adversos comunes son menores y transitorios (16). Sin embargo, dado que especialmente la cloroquina puede ser cardiotóxica y neurotóxica, estos medicamentos deben ser usados con precaución y debe evitarse la automedicación (16).

En todo el mundo los investigadores están estudiando la actividad de otros medicamentos contra el SARS-CoV-2, como el lopinavir, el ritonavir, la ribavirina, el penciclovir, la nitazoxanida y el nafamostat. Sin embargo, a diferencia de la cloroquina, dichos fármacos no están ampliamente disponibles para el tratamiento de un número elevado de pacientes con COVID-19. Por lo tanto, de todos estos medicamentos la cloroquina parece el medicamento de mayor potencial para el uso a gran escala debido a su disponibilidad, seguridad y bajo costo (26). La FDA ya emitió una "autorización de uso de emergencia" para el fosfato de cloroquina y el sulfato de hidroxicloroquina (29), la primera autorización de este tipo emitida en Estados Unidos. Compañías como Sandoz y Novartis donaron 30 millones de dosis para la lucha contra la COVID-19. Además, otras compañías han redoblado la producción para cubrir la creciente demanda en Estados Unidos y otros países (30).

\section{Limitaciones}

Debido al poco tiempo de preparación y la urgencia de los resultados, la estrategia de búsqueda de la presente revisión no contó con la orientación de un bibliotecólogo. Sin embargo, nuestro equipo tiene experiencia en la elaboración de revisiones sistemáticas (31-36). Solo se incluyeron tres bases de datos, pero cada una de ellas aporta un aspecto clave a la búsqueda: Embase está especializada en farmacología, PubMed es la base de datos médica más nutrida del mundo, y Lilacs es la base de datos latinoamericana más completa. No incluimos documentos exclusivamente en mandarín, pero incluimos estudios en cinco idiomas.

La revisión no incluyó una evaluación de la calidad de los datos, pues las revisiones sistemáticas exploratorias, por lo general, no incluyen este tipo de evaluaciones, ya que a diferencia de las revisiones sistemáticas clásicas, la pregunta de investigación es menos específica (18). Además, este tipo de estudios reporta la evidencia independientemente de su calidad (37). La extensión de la declaración PRISMA para el reporte de revisiones sistemáticas exploratorias (23), la cual se siguió en este estudio, desaconseja realizar análisis de la calidad de la información. Por último, los hallazgos que se presentan reflejan la escasa información disponible y el deficiente diseño de los estudios encontrados. 


\section{Conclusiones}

La utilización de medicamentos conocidos para enfrentar la COVID-19 es una estrategia práctica porque ya se cuenta con información sobre los efectos adversos, la posología y las interacciones farmacológicas. A la luz de la información disponible, se recomienda el uso de la cloroquina y la hidroxicloroquina para el tratamiento de pacientes con COVID-19 única y exclusivamente bajo autorización y vigilancia médica en ensayos controlados aleatorizados. El uso de estos medicamentos por fuera del ámbito clínico puede ocasionar reacciones adversas potencialmente fatales, por lo que su venta sin vigilancia y su uso indiscriminado deben controlarse estrictamente.

Hasta el momento, solo un ensayo clínico no aleatorizado con un reducido tamaño de muestra e importantes deficiencias, ha reportado que la hidroxicloroquina es efectiva para eliminar el SARS-CoV-2 en seres humanos. Es urgente el acceso a análisis interinos de otros ensayos clínicos para determinar la efectividad y la seguridad de la cloroquina y la hidroxicloroquina en pacientes con COVID-19. Se sabe de por lo menos dos ensayos clínicos cuyos resultados están previstos para el 2020.

Además de averiguar el perfil de seguridad y la efectividad de los medicamentos para controlar la COVID-19, es importante saber si es posible recomendar la cloroquina o la hidroxicloroquina como quimioprofilaxis para los profesionales de la salud que se infectan en sus labores y mueren por esta enfermedad. También debe determinarse si el efecto clínico depende de la edad de los pacientes, la presentación clínica o la etapa de la enfermedad. Estas son preguntas clave que deberán ser respondidas en futuros estudios clínicos.

\section{Agradecimientos}

A Cassandra Laurie del Department of Epidemiology, Biostatistics and Occupational Health de la McGill University, que contribuyó en la recolección de datos y prestó asesoría en virología. La investigadora expresó su aprobación para aparecer en los agradecimientos.

\section{Referencias}

1. World Health Organization. WHO Director-General's opening remarks at the media briefing on COVID-19. Fecha de consulta: 23 de marzo de 2020. Disponible en: https://www. who.int/ $\mathrm{dg} / \mathrm{speeches/detail/who-director-general-s-opening-remarks-at-the-media-briefing-on-covid-}$ 19---11-march-2020

2. World Health Organization. Coronavirus disease 2019. Fecha de consulta: 8 de abril de 2020. Disponible en: https://www.who.int/emergencies/diseases/novel-coronavirus-2019

3. Savarino A, Di Trani L, Donatelli I, Cauda R, Cassone A. New insights into the antiviral effects of chloroquine. Lancet Infect Dis. 2006;6:67-9. https://doi.org/10.1016/S1473-3099(06)70361-9

4. Ksiazek TG, Erdman D, Goldsmith CS, Zaki SR, Peret T, Emery S, et al. A novel coronavirus associated with severe acute respiratory syndrome. N Engl J Med. 2003;348:1953-66. https://doi.org/10.1056/NEJMoa030781

5. Keyaerts E, Vijgen L, Maes P, Neyts J, Ranst M Van. In vitro inhibition of severe acute respiratory syndrome coronavirus by chloroquine. Biochem Biophys Res Commun. 2004;323:264-8. https://doi.org/10.1016/j.bbrc.2004.08.085

6. Yu IT-S, Qiu H, Tse LA, Wong TW. Severe acute respiratory syndrome beyond Amoy gardens: Completing the incomplete legacy. Clin Infect Dis. 2014;58:683-6. https://doi.org/10.1093/cid/cit797

7. Colson P, Rolain J-M, Raoult D. Chloroquine for the 2019 novel coronavirus SARS-CoV-2. Int J Antimicrob Agents. 2020;55:105923. https://doi.org/10.1016/j.jjantimicag.2020.105923 
8. Keyaerts E, Li S, Vijgen L, Rysman E, Verbeeck J, Van Ranst M, et al. Antiviral activity of chloroquine against human coronavirus OC43 infection in newborn mice. Antimicrob Agents Chemother. 2009;53:3416-21. https://doi.org/10.1128/AAC.01509-08

9. de Wilde AH, Jochmans D, Posthuma CC, Zevenhoven-Dobbe JC, van Nieuwkoop S, Bestebroer TM, et al. Screening of an FDA-approved compound library identifies four smallmolecule inhibitors of Middle East respiratory syndrome coronavirus replication in cell culture. Antimicrob Agents Chemother. 2014;58:4875-84. https://doi.org/10.1128/AAC.03011-14

10. Rolain J-M, Colson P, Raoult D. Recycling of chloroquine and its hydroxyl analogue to face bacterial, fungal and viral infections in the 21st century. Int J Antimicrob Agents. 2007;30:297308. https://doi.org/10.1016/j.ijantimicag.2007.05.015

11. Wang M, Cao R, Zhang L, Yang X, Liu J, Xu M, et al. Remdesivir and chloroquine effectively inhibit the recently emerged novel coronavirus (2019-nCoV) in vitro. Cell Res. 2020;30:26971. https://doi.org/10.1038/s41422-020-0282-0

12. Devaux CA, Rolain J-M, Colson P, Raoult D. New insights on the antiviral effects of chloroquine against coronavirus: What to expect for COVID-19? Int J Antimicrob Agents. 2020;105938. https://doi.org/10.1016/j.ijantimicag.2020.105938

13. Huang C, Wang Y, Li X, Ren L, Zhao J, Hu Y, et al. Clinical features of patients infected with 2019 novel coronavirus in Wuhan, China. Lancet. 2020;395:497-506. https://doi.org/10.1016/S0140-6736(20)30183-5

14. Shang L, Zhao J, Hu Y, Du R, Cao B. On the use of corticosteroids for 2019-nCoV pneumonia. Lancet. 2020;395:683-4. https://doi.org/10.1016/S0140-6736(20)30361-5

15. Ministerio de Salud y Protección Social - República de Colombia. Hidroxicloroquina y cloroquina se podrán usar para tratamiento de covid - 19. 2020. Fecha de consulta: 8 de abril de 2020. Disponible en: https://www.minsalud.gov.co/Paginas/Hidroxicloroquina-ycloroquina-se-podran-usar-para-tratamiento-de-covid---19.aspx

16. Touret F, de Lamballerie X. Of chloroquine and COVID-19. Antiviral Res. 2020;177:104762. https://doi.org/10.1016/j.antiviral.2020.104762

17. Song P, Karako T. COVID-19: Real-time dissemination of scientific information to fight a public health emergency of international concern. Biosci Trends. 2020;14:1-2. https://doi.org/10.5582/bst.2020.01056

18. Arksey H, O'Malley L. Scoping studies: Towards a methodological framework. Int J Soc Res Methodol. 2005;8:19-32. https://doi.org/10.1093/geront/gnz021

19. Levac D, Colquhoun H, O'Brien KK. Scoping studies: Advancing the methodology. Implement Sci. 2010;5:69. https://doi.org/10.1186/1748-5908-5-69

20. World Health Organization. WHO Registry Network. 2020. Fecha de consulta: 25 de marzo de 2020. Disponible en: https://www.who.int/ictrp/network/primary/en/

21. Ouzzani M, Hammady H, Fedorowicz Z, Elmagarmid A. Rayyan-a web and mobile app for systematic reviews. Syst Rev. 2016;5:210. https://doi.org/10.1186/s13643-016-0384-4

22. Grudniewicz A, Nelson M, Kuluski K, Lui V, Cunningham H V., X Nie J, et al. Treatment goal setting for complex patients: protocol for a scoping review. BMJ Open. 2016;6:e011869. https://doi.org/10.1136/bmjopen-2016-011869

23. Tricco AC, Lillie E, Zarin W, O'Brien KK, Colquhoun H, Levac D, et al. PRISMA Extension for Scoping Reviews (PRISMA-ScR): Checklist and Explanation. Ann Intern Med. 2018;169:467. https://doi.org/10.7326/M18-0850

24. Gao J, Tian Z, Yang X. Breakthrough: Chloroquine phosphate has shown apparent efficacy in treatment of COVID-19 associated pneumonia in clinical studies. Biosci Trends. 2020;14:7273. https://doi.org/10.5582/bst.2020.01047

25. Yao X, Ye F, Zhang M, Cui C, Huang B, Niu P, et al. In vitro antiviral activity and projection of optimized dosing design of hydroxychloroquine for the treatment of severe acute respiratory syndrome coronavirus 2 (SARS-CoV-2). Clin Infect Dis. 2020. https://doi.org/10.1093/cid/ciaa237

26. Liu J, Cao R, Xu M, Wang X, Zhang $\mathrm{H}, \mathrm{Hu} \mathrm{H}$, et al. Hydroxychloroquine, a less toxic derivative of chloroquine, is effective in inhibiting SARS-CoV-2 infection in vitro. Cell Discov. 2020;6:16. https://doi.org/10.1038/s41421-020-0156-0

27. Gautret P, Lagier J-C, Parola P, Hoang VT, Meddeb L, Mailhe M, et al. Hydroxychloroquine and azithromycin as a treatment of COVID-19: Results of an open-label non-randomized clinical trial. Int J Antimicrob Agents. 2020;105949. https://doi.org/10.1016/j.ijantimicag.2020.105949 
28. Wilson P. COVID-19, hydroxychloroquine, and the death of evidence-based medicine. The Methods Man. 2020. Fecha de consulta: 2 de abril de 2020. Disponible en: https://www. methodsman.com/blog/covid-19-evidence

29. Hinton DM. US Food \& Drug Administration Emergency Use Autorization for chloroquine phosphate and hydroxychloroquine sulfate use for the treatment of COVID-19. 2020. p. 1-8. Fecha de consulta: 2 de abril de 2020. Disponible en: https://dailymed.nlm.nih.gov/dailymed/ druglnfo.cfm? setid=f398f8a9-92f3-47cb-81c2-6078806a464d

30. U.S. Department of Health and Human Services (HHS). HHS accepts donations of medicine to Strategic National Stockpile as possible treatments for COVID-19 patients I HHS.gov. News. 2020. Fecha de consulta: 2 de abril de 2020. Disponible en: https://www.hhs.gov/ about/news/2020/03/29/hhs-accepts-donations-of-medicine-to-strategic-national-stockpileas-possible-treatments-for-covid-19-patients.html\#

31. O'Brien N, Hong QN, Law S, Massoud S, Carter A, Kaida A, et al. Health system features that enhance access to comprehensive primary care for women living with HIV in highincome settings: A systematic mixed studies review. AIDS Patient Care STDS. 2018;32:12948. https://doi.org/10.1089/apc.2017.0305

32. Alvarado-Castro V, Paredes-Solís S, Nava-Aguilera E, Morales-Pérez A, Alarcón-Morales L, Balderas-Vargas NA, et al. Assessing the effects of interventions for Aedes aegypti control: Systematic review and meta-analysis of cluster randomised controlled trials. BMC Public Health. 2017;17(Supl.1):384. https://doi.org/10.1186/s12889-017-4290-z

33. Shalabi D, Mitchell S, Andersson N. Review of gender violence among arab immigrants in Canada: Key issues for prevention efforts. J Fam Violence. 2015;30:817-25. https://doi.org/10.1007/s10896-015-9718-6

34. Shea BJ, Grimshaw JM, Wells GA, Boers M, Andersson N, Hamel C, et al. Development of AMSTAR: A measurement tool to assess the methodological quality of systematic reviews. BMC Med Res Methodol. 2007;7. https://doi.org/10.1186/1471-2288-7-10

35. Pimentel J, Arias A, Ramírez D, Molina A, Chomat A-M, Cockcroft A, et al. Game-based learning interventions to foster cross-cultural care training: A scoping review. Games Health J. 2020. https://doi.org/10.1089/g4h.2019.0078

36. Pimentel J, Ansari U, Omer K, Gidado Y, Baba MC, Andersson N, et al. Factors associated with short birth interval in low- and middle-income countries: A systematic review. BMC Pregnancy Childbirth. 2020;20:156. https://doi.org/10.1186/s12884-020-2852-z

37. Peters MDJ, Godfrey CM, Khalil H, Mclnerney P, Parker D, Soares CB. Guidance for conducting systematic scoping reviews. Int J Evid Based Healthc. 2015;13:141-6. https://doi.org/10.1097/XEB.0000000000000050 


\section{Archivo suplementario 1}

1. Estrategia de búsqueda (última actualización, marzo 25 de 2020)

\begin{tabular}{l}
\hline Pubmed \\
\hline (((((Coronavirus[Title]) OR COVID[Title]) OR SARS-CoV-2[Title/Abstract]) OR “severe acute respiratory \\
syndrome coronavirus 2" [Supplementary Concept]) OR "COVID-19" [Supplementary Concept])) AND \\
(((“Aminoquinolines”[Mesh]) OR chloroquine[Title]) OR hydroxychloroquine[Title]) \\
\hline Embase \\
\hline 1. exp SARS coronavirus/ or exp Coronavirinae/ \\
2. exp aminoquinoline derivative/ \\
3. exp chloroquine/ \\
4. hydroxychloroquine/ \\
5. 2 or 3 or 4 \\
6. 1 and 5 \\
\hline Lilacs \\
(“Covid" OR "Coronavirus" OR "SARS-CoV-2" OR "Virus del SARS”) AND (Aminoquinolinas OR \\
Cloroquina OR Hidroxicloroquina) \\
\hline
\end{tabular}

2. Número de ensayos clínicos incluidos en cada base de datos (búsqueda, marzo 23, 2020)

\begin{tabular}{llc}
\hline Nombre & Sitio Web & Incluidos \\
\hline U.S. National Library of Medicine & clinicaltrials.gov & 8 \\
Australian New Zealand Clinical Trials Registry (ANZCTR) & anzctr.org.au & 0 \\
Brazilian Clinical Trials Registry (ReBec) & ensaiosclinicos.gov.br & 0 \\
Chinese Clinical Trial Registry & chictr.org.cn & 14 \\
Clinical Research Information Service (CRiS), Republic of Korea & cris.nih.go.kr & 0 \\
Clinical Trials Registry - India (CTRI) & ctri.nic.in/Clinicaltrials/advsearch.php & 0 \\
Cuban Public Registry of Clinical Trials(RPCEC) & registroclinico.sld.cu/en/home & 0 \\
EU Clinical Trials Register (EU-CTR) & clinicaltrialsregister.eu & 0 \\
German Clinical Trials Register (DRKS) & drks.de/drks_web/ & 0 \\
Iranian Registry of Clinical Trials (IRCT) & irct.ir & 0 \\
ISRCTN & isrctn.com/ & 1 \\
Japan Primary Registries Network (JPRN) & rctportal.niph.go.jp/ & 1 \\
The Netherlands National Trial Register (NTR) & trialregister.nl/ & 0 \\
Pan African Clinical Trial Registry (PACTR) & pactr.samrc.ac.za/ & 0 \\
Peruvian Clinical Trial Registry (REPEC) & ensayosclinicos-repec.ins.gob.pe/ & 0 \\
\hline
\end{tabular}

Los términos de búsqueda en cada base de datos fueron: (Aminoquinolinas OR Cloroquina OR Hidroxicloroquina) 


\section{Archivo suplementario 2}

PRISMA Extension for Scoping reviews (PRISMA-ScR) 2018 Checklist $^{1}$

\begin{tabular}{|c|c|c|c|}
\hline Section/topic & $\#$ & PRISMA-ScR Checklist item & $\begin{array}{l}\text { Reported on } \\
\text { page \# }\end{array}$ \\
\hline \multicolumn{4}{|l|}{ TITLE } \\
\hline Title & 1 & Identify the report as a scoping review. & 1 \\
\hline
\end{tabular}

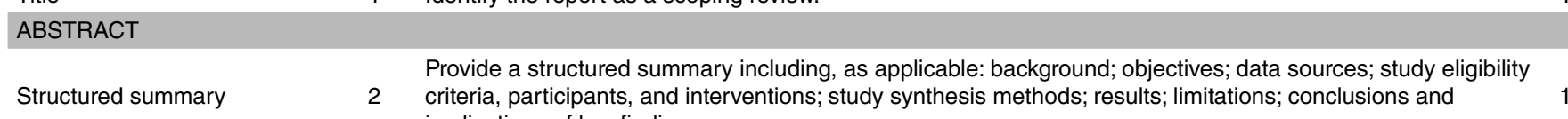
implications of key findings.

\section{INTRODUCTION}

Rationale

3

Describe the rationale for the review in the context of what is already known. Explain why the review question(s)/objective(s) lend themselves to a scoping review approach.

Provide an explicit statement of the question(s) and objective(s) being addressed with reference to their key

Objectives conceptualize the review question(s) and/or objective(s)).

\section{METHODS}

Protocol and registration

5 Indicate if a review protocol exists, if and where it can be accessed (e.g., Web address), and, if available, provide registration information including registration number.

Eligibility criteria

6 Specify the characteristics of the sources of evidence

Information sources

Describe all information sources (e.g., databases with dates of coverage, contact with study authors to identify additional sources) in the search and date last searched.

Search

Present full elect

Selection of sources of evidence 9

Data charting process epeated.

State the process for selecting studies (i.e., screening, eligibility) included in the scoping review.

Describe the methods of charting data from the included sources of evidence (e.g. piloted forms; forms that

10 have been tested by the team before their use, whether data charting was done independently, in duplicate) and any processes for obtaining and confirming data from investigators.

Data items

11 List and define all variables for which data were sought and any assumptions and simplifications made.

Critical appraisal of individual sources of evidence

Summary measures If done, provide a rationale for conducting a critical appraisal of included sources of evidence; describe the methods used and how this information was used in any data synthesis (if appropriate).

13 Not applicable for scoping reviews.

Synthesis of results

Risk of bias across studies

Describe the methods of handling and summarizing the data that were charted.

Additional analyses

16 Not applicable for scoping reviews.

\section{RESULTS}

Selection of sources of evidence 17

Characteristics of sources of evidence

Critical appraisal within sources of evidence

Results of individual sources of evidence

Synthesis of results

Risk of bias across studies

Additional analysis

Give numbers of studies screened, assessed for eligibility, and included in the review, with reasons for exclusions at each stage, ideally using a flow diagram.

18 For each source of evidence, present characteristics for which data were charted and provide the citations.

19 If done, present data on critical appraisal of included sources of evidence (see item 12).

3-4

Archivo suplementario 1

4

4

4

NA

NA

4

NA

NA

20 For each included source of evidence, present the relevant data that were charted that relate to the review question(s) and objective(s).

21 Summarize and/or present the charting results as they relate to the review question(s) and objective(s).

5-10

22 Not applicable for scoping reviews.

NA

23 Not applicable for scoping reviews.

Figura 1

Tablas 1 y 2

NA

Tablas 1 y 2

A

NA

\section{DISCUSSION}

\section{Summary of evidence}

Limitations

Conclusions

\section{FUNDING}

\section{4}

25

26

Summarize the main results (including an overview of concepts, themes, and types of evidence available), explain how they relate to the review question(s) and objectives, and consider the relevance to key groups

Provide a general interpretation of the results with respect to the review question(s) and objective(s), as well as potential implications and/or next steps.

12

27 Describe sources of funding for the included sources of evidence, as well as sources of funding for the scoping review. Describe the role of the funders of the scoping review. 